\title{
Physikalischer Modelversuch und Cfd-Simulation einer asymmetrischen Drossel in einem T-Abzweigstück
}

Zusammenfassung: Zur Ermittlung der Verluste einer asymmetrischen Drossel, die in einem T-förmigen Abzweigstück des Triebwasserweges einer Hochdruckanlage situiert ist, wurden hydraulische Modellversuche im Maßstab 1:21 und numerische Simulationen unter unterschiedlichen Strömungsverhältnissen durchgeführt. Aufgrund der komplexen Form des Abzweigstückes wurde die Form mit einer neuartigen Methode mittels CNC-Technik aus einem Hartschaumblock gefräst. Die CFD-Simulationen wurden durchgeführt um zu ermitteln, unter welchen Umständen die Numerische Simulation verlässliche Ergebnisse liefern kann.

Physical scale model test and CFD simulation for an asymmetrical throttle in a T-branch

Summary: Hydraulic model tests at a scale of 1:21 and numerical simulations were conducted for various flow conditions to study the loss occurring in an asymmetrical throttle situated in a T-shaped branch in the power conduit of a highhead hydro plant. The complex shape of the piece had to be cut from a rigid foam block using CNC. The intended purpose of the CFD simulations was to study the circumstances under which numerical simulations can yield reliable results.

\section{Einführung}

Die asymmetrische Drossel befindet sich in dem abzweigenden Ast eines T-förmigen Abzweigstückes im Druckschacht einer Hochdruck-Wasserkraftanlage. Der kreisförmige Schacht (Druck- und Steigschacht) besitzt einen Innendurchmesser von 3,60 m (Zweig , a') und eine Neigung von $42^{\circ}$ gegen die Horizontale. An den Druckschacht schließt ein Krümmer an, dessen kreisförmiger Querschnitt sich auf 7,20 m (Zweig , c') aufweitet und dann in die hufeisenförmige Wasserschloss-Unterkammer übergeht. Gegen Ende des Krümmers, ca. $5 \mathrm{~m}$ vor dem Beginn des Übergangstückes, zweigt im rechten Win-

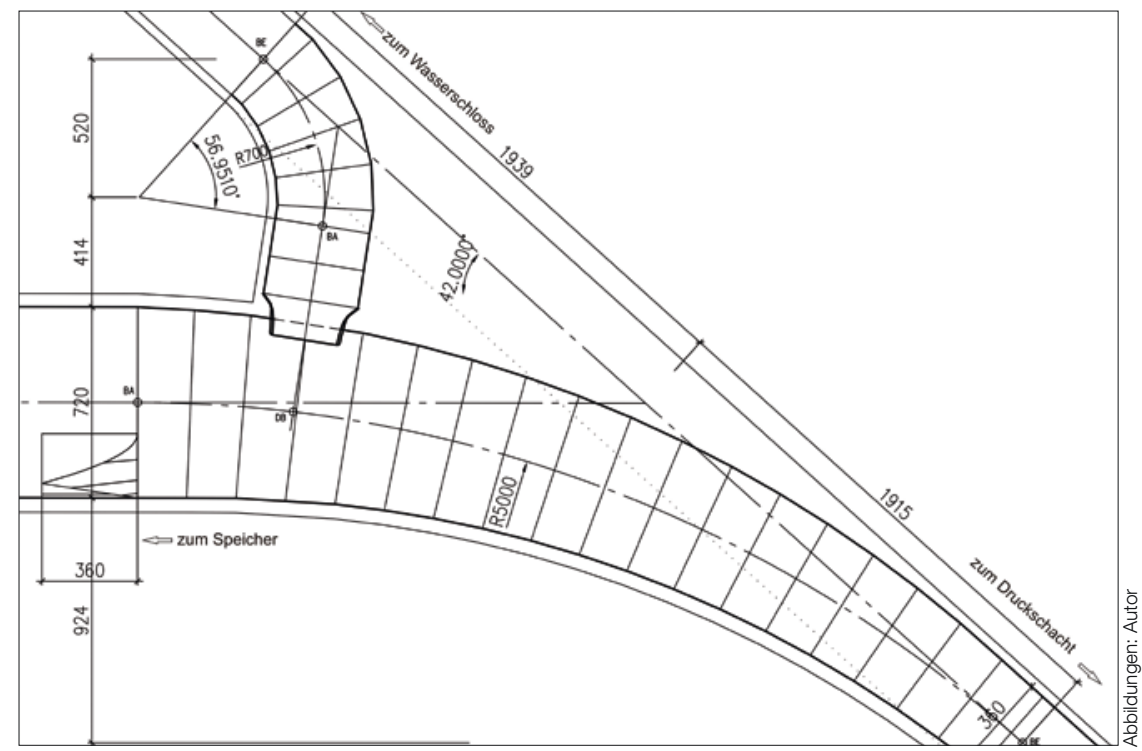

Abb. 1: Vertikalschnitt durch das T-Stück (Naturmaße; in cm).

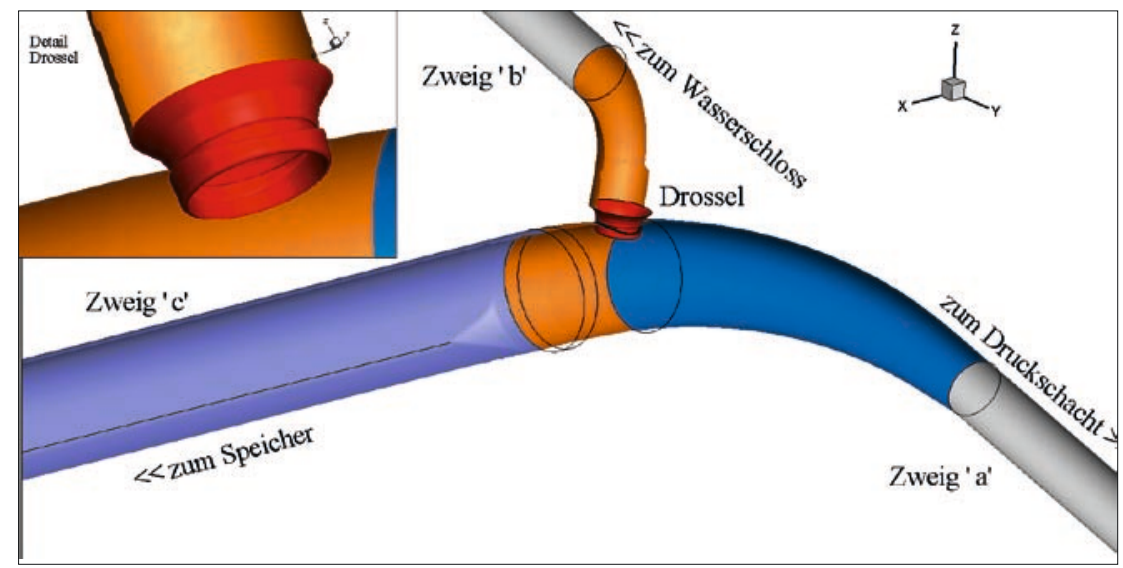

Abb. 2: 3d-Illustration von Drossel und Krümmer mit T-Abzweigstück.
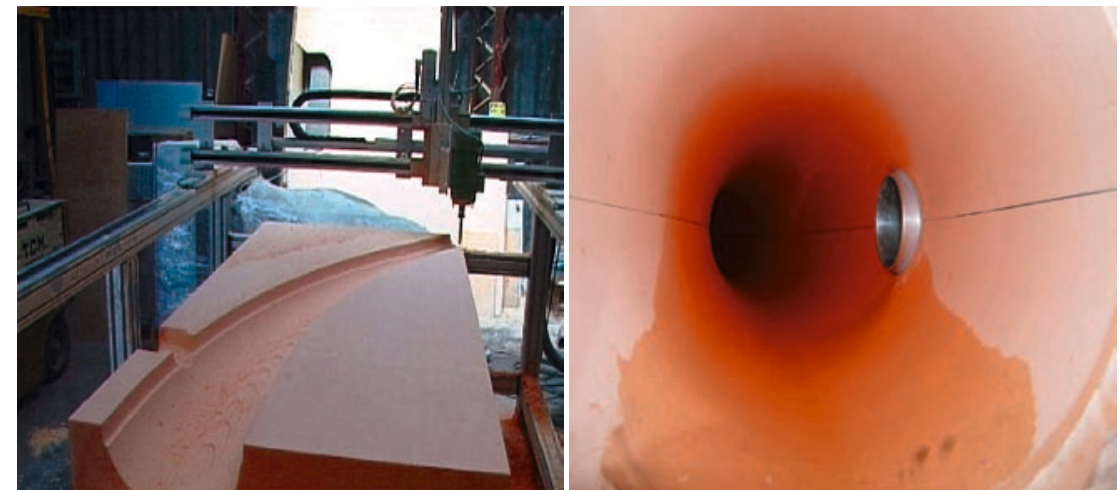

Abb. 3 a, b: Fräsen der Krümmer-Halbschalen (links); Drossel (rechts). 
kel zur Krümmerachse der Übergangsbogen zum Steigschacht ab (Zweig , $\mathbf{b}^{\prime}$ ), in dem sich die Drossel befindet.

Abbildung 2 zeigt eine 3d-Illustration der Drossel und des gebogenen Krümmers mit dem T-Stück.

\subsection{Untersuchte Betriebsfälle}

Sieben verschiedene Betriebsfälle wurden untersucht (vgl. Abb. 2 und Tab. 1).

\section{MODELLVERSUCHE}

Eine physikalisch exakte Nachbildung nach dem Reynolds'schen Modellgesetz ist bei solchen Modellversuchen in der Regel unmöglich, da sich unter Zugrundelegung des Reynoldschen Modellgesetzes das Verhältnis der Geschwindigkeiten in Natur und Modell im reziproken Längenmaßstab verhält (bei gleicher Zähigkeit). Man hilft sich in der Praxis damit, den Durchfluss bis zum versuchstechnisch Möglichen zu steigern und bei unterschiedlichen Durchflüssen die Verlustbeiwerte zu messen (vgl. z. B. Kobus, 1978). Nähert sich mit zunehmenden Reynoldszahlen der Verlustbeiwert - innerhalb gewisser messtechnischer und versuchstechnischer Grenzen - einem konstanten Wert an, so kann er auf den entsprechenden Reynoldsbereich in der Natur extrapoliert werden.

Der Längenmaßstab wurde mit 1:21 festgelegt und das Gesamtsystem - wie bei solchen Versuchen häufig angewandt - horizontal liegend eingebaut (der Einfluss der Gravitation ist hier vernachlässigbar). Die Herstellung des Krümmers erfolgte durch Fräsen: mittels CNC-Technik wurden aus 2 Hartschaumblöcken a $2 \times 1$ x 0,5 m zwei Halbschalen gefräst, danach händisch glatt geschliffen und dann entlang der Symmetrieebene zusammengespannt (vgl. Abb. 3a).

Die Drossel wurde aus einem Aluminiumblock ebenfalls mittels CNC-Technik gefräst, in den vorbereiteten Rohrabzweiger eingebaut und am Hartschaumblock befestigt ( $v g l . A b b .3 b)$.

Die anschließenden Rohrleitungen und der Rohrkrümmer bestanden aus Plexiglas (Rohrdurchmesser $172 \mathrm{~mm}$, die hufeisenförmige Wasserschloss-Unterkammer hatte einen mittleren Durchmesser von ca. $348 \mathrm{~mm}$ ). Die Rohrleitungen wurden ebenfalls zugfest am Krümmerblock befestigt. An den Enden der Rohräste waren Honeycombs zur Strömungsgleichrichtung eingebaut. Je nach untersuchtem Lastfall wurde durch

\begin{tabular}{lllll}
$\begin{array}{l}\text { TABELLE } 1 \\
\text { Betriebsfälle }\end{array}$ & & \\
Fall & Beschreibung & Zweig 'a' & Zweig 'b' & Zweig 'c' \\
\hline 1 & $\begin{array}{l}100 \% \text { Zufluss vom Speicher } \\
100 \% \text { zum Wasserschloss }\end{array}$ & 0 & -- & ++ \\
\hline 2 & $\begin{array}{l}100 \% \text { Zufluss vom Speicher } \\
50 \% \text { zum Wasserschloss, } 50 \% \text { zum Druckschacht }\end{array}$ & - & - & ++ \\
\hline 3 & $\begin{array}{l}100 \% \text { Zufluss vom Druckschacht } \\
100 \% \text { zum Wasserschloss }\end{array}$ & ++ & -- & 0 \\
\hline 4 & $\begin{array}{l}100 \% \text { Zufluss vom Druckschacht } \\
50 \% \text { zum Wasserschloss, } 50 \% \text { zum Speicher }\end{array}$ & ++ & - & - \\
\hline 5 & $\begin{array}{l}100 \% \text { Zufluss vom Wasserschloss } \\
100 \% \text { zum Speicher }\end{array}$ & 0 & ++ & -- \\
\hline 6 & $\begin{array}{l}100 \% \text { Zufluss vom Wasserschloss } \\
100 \% \text { zum Druckschacht }\end{array}$ & -- & ++ & 0 \\
\hline 7 & $\begin{array}{l}100 \% \text { Zufluss vom Wasserschloss } \\
50 \% \text { zum Speicher, } 50 \% \text { zum Druckschacht }\end{array}$ & - & ++ & - \\
\hline++ Zufluss $100 \%,--$ Abfluss $100 \%$, - Abfluss $50 \%, 0$ kein Durchfluss & & \\
\hline
\end{tabular}

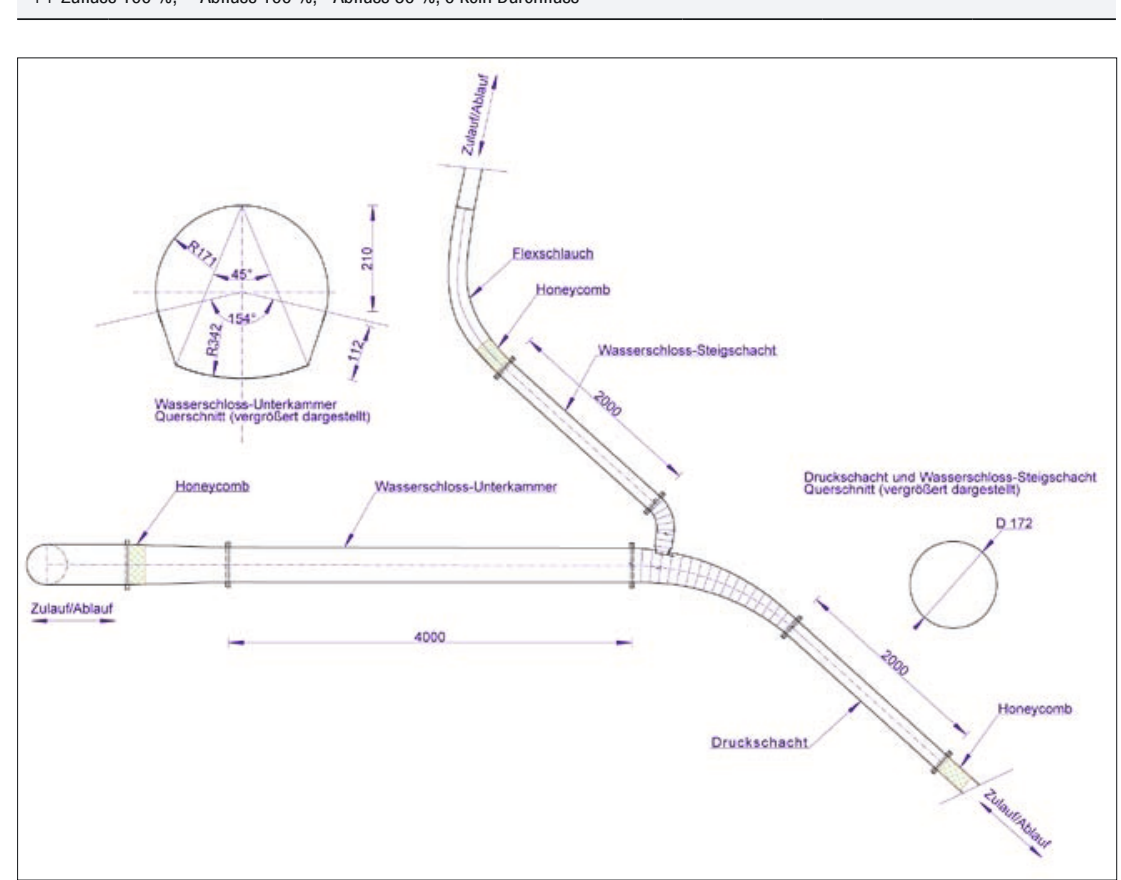

Abb. 4: Skizze des Modellstandes (Maße in $\mathrm{mm}$ ).

TABELLE 2

Verlustbeiwerte aus den Modellversuchen

\begin{tabular}{llll} 
Fall & $\mathbf{Q}_{\mathbf{D r}} / \mathbf{Q}_{\text {total }}$ & $\begin{array}{l}\mathbf{K}_{2-6} \mathbf{b z w} . \mathbf{K}_{5-1} \\
\text { (nicht durch Drossel gehend) }\end{array}$ & $\begin{array}{l}\mathbf{K}_{2-4} \mathbf{b z W} . \mathbf{K}_{3-1} \\
\text { (durch Drossel) }\end{array}$ \\
\hline Fall 1 & 1,0 & $\zeta_{5-1}=0,03$ & $\zeta_{5-4}=1,60$ \\
\hline Fall 2 & 0,5 & $\zeta_{5-1}=0,05$ & $\zeta_{5-4}=1,63$ \\
\hline Fall 3 & 1,0 & $\zeta_{2-6}=0,07$ & $\zeta_{2-4}=1,72$ \\
\hline Fall 4* & 0,5 & $\zeta_{2-6}=0,23$ & $\zeta_{2-4}=2,20$ \\
\hline Fall 5 & 1,0 & & $\zeta_{3-6}=1,00, \zeta_{3-1}=0,97$ \\
\hline Fall 6 & 1,0 & & $\zeta_{3-6}=0,95, \zeta_{3-1}=1,04$ \\
\hline Fall 7 & 0,5 & & $\zeta_{3-6}=1,04, \zeta_{3-1}=1,06$ \\
\hline
\end{tabular}

* Im Gegensatz zu Lastfall 3 wird im Lastfall 4 beim selben Durchfluss durch die Drossel der Strang a mit der doppelten Wassermenge (und Fließgeschwindigkeit) durchströmt. Somit ist die Geschwindigkeitshöhe im Strang a im LF4 wesentlich größer, als beim selben Drosseldurchfluss im LF3, während die gemessenen statischen Drücke annähernd gleich sind. Daher ist auch der aus dem totalen Druck errechnete Verlustbeiwert im LF4 deutlich größer. 


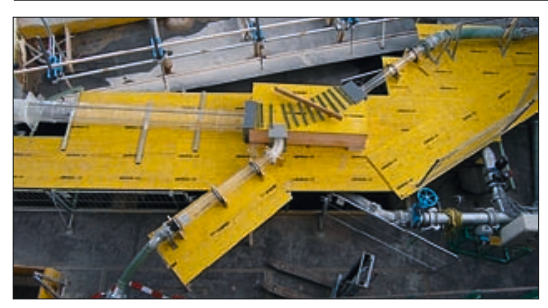

Abb. 5: Foto des Versuchsstandes.
Steuerung der Schieber in den Ablaufrohrsträngen die entsprechende Durchflusseinstellung vorgenommen und der Druck so eingestellt, dass einerseits der Druck in den Messsstellen ausreichend groß war, um ein Lufteinziehen zu verhindern und andererseits nicht zu hoch wurde, um ein Bersten der Leitung zu verhindern.

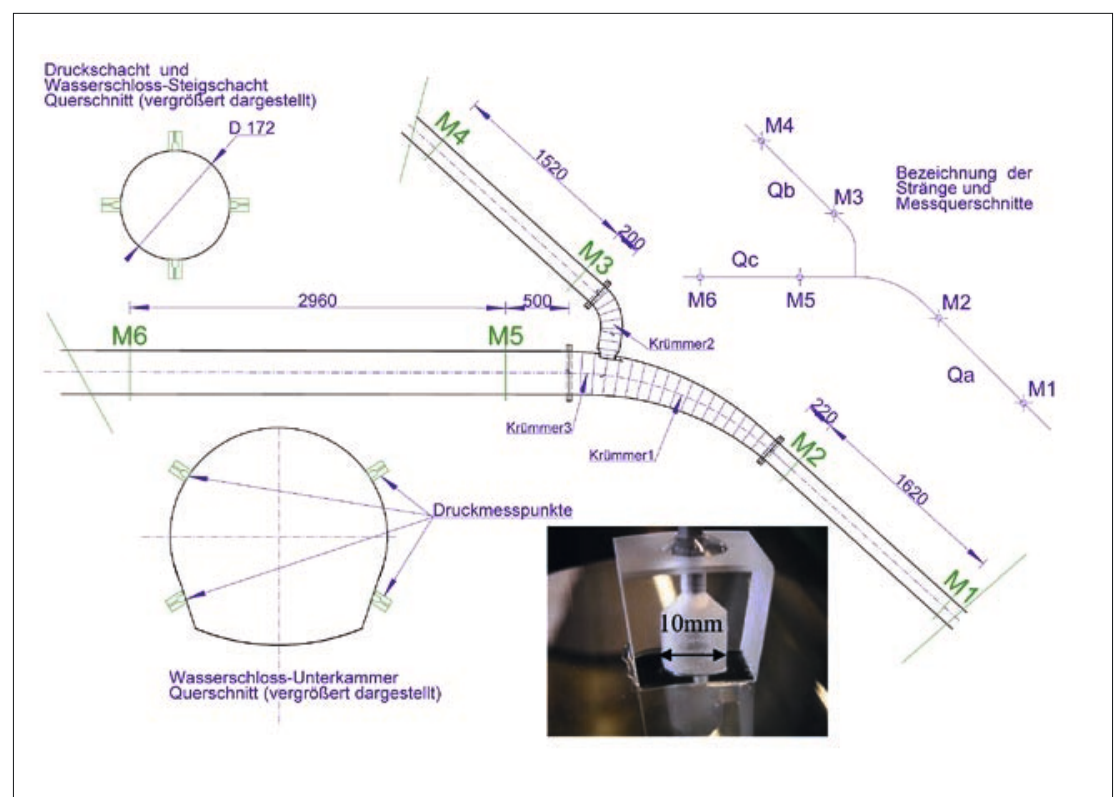

Abb. 6: Anordnung der Druckmesspunkte (Maße in mm).

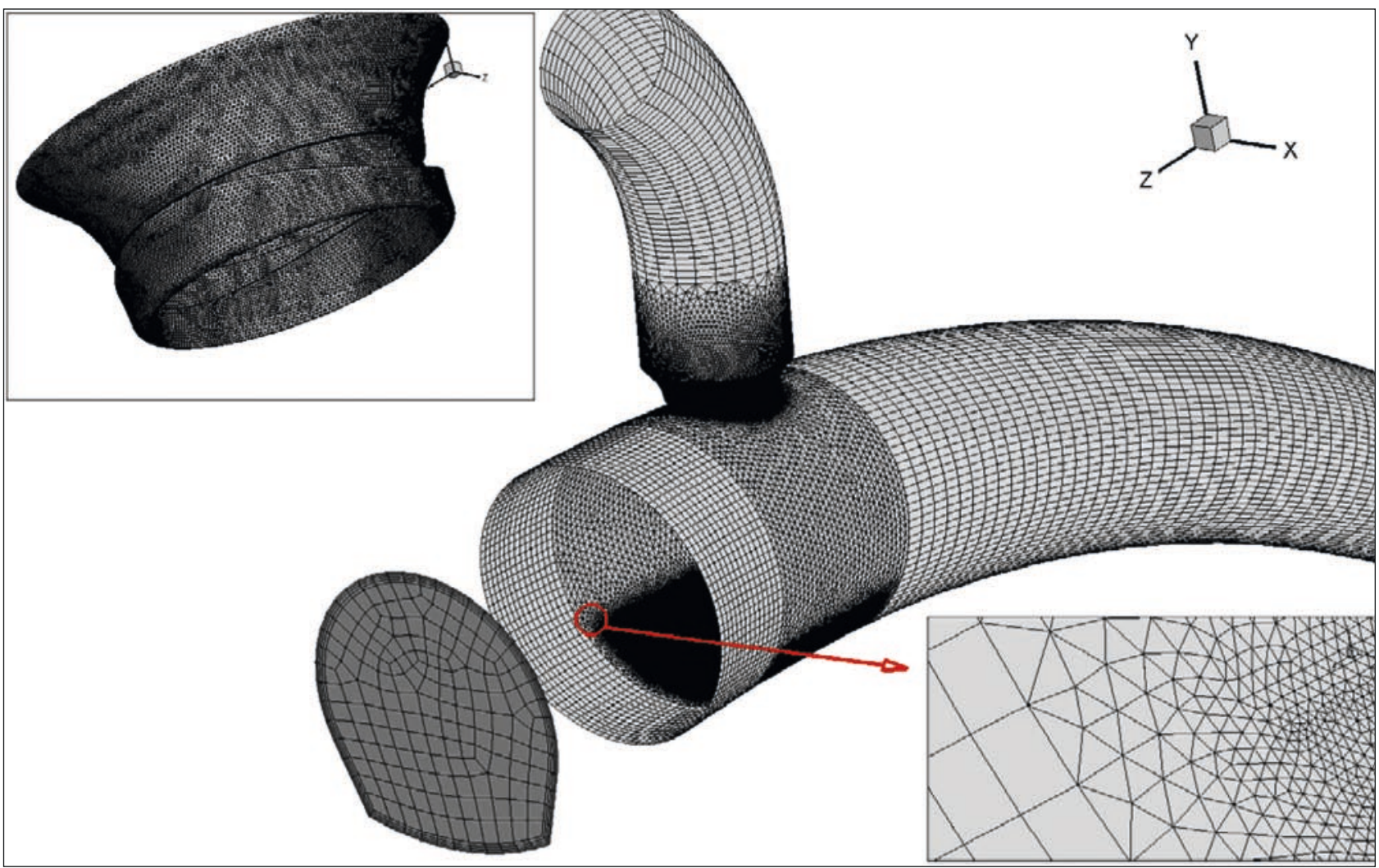

Abb. 7: Netzdetail.

Die Durchflussmessung erfolgte mit 2 magnetisch-induktiven Durchflussmessern (IDM), von denen je einer in der $\mathrm{Zu} /$ Ablaufrohrleitung des Druckund des Wasserschloss-Steigschachtes angeordnet war. Druckdifferenzen zwischen zwei Messquerschnitten wurden mit einem Differenzdruckaufnehmer gemessen.

Zur Druckmessung waren zwei Messquerschnitte in jedem Zweig vorgesehen: der erste in Fließrichtung möglichst nahe vor dem T-Stück und der zweite nach dem T-Stück möglichst weit davon entfernt (mehr als $10 \mathrm{xD}$ ), sodass sich die Druckverhältnisse dort wieder weitgehend vergleichmäßigt hatten.

\subsection{Ermittlung der Drosselverluste}

Der Verlustbeiwert errechnet sich aus:

$\zeta_{\mathrm{ij}}=\frac{\begin{array}{c}\text { totaler Druck in } \\ \text { Querschnitt i }\end{array}-\begin{array}{c}\text { totaler Druck in } \\ \text { Querschnitt j }\end{array}-\begin{array}{c}\text { Rohr- } \\ \text { reibung }\end{array}}{\mathrm{v}_{\mathrm{Dr}}^{2} / 2 \mathrm{~g}}$

mit $v_{D r} \ldots$ mittlere Fließgeschwindigkeit im engsten Drosselquerschnitt $\left(D_{D r}=2,47 \mathrm{~m}\right.$ in Natur / 0,117 m im Modell). Die mittleren örtlichen Geschwindigkeitshöhen wurden zu den gemittelten (statischen) 


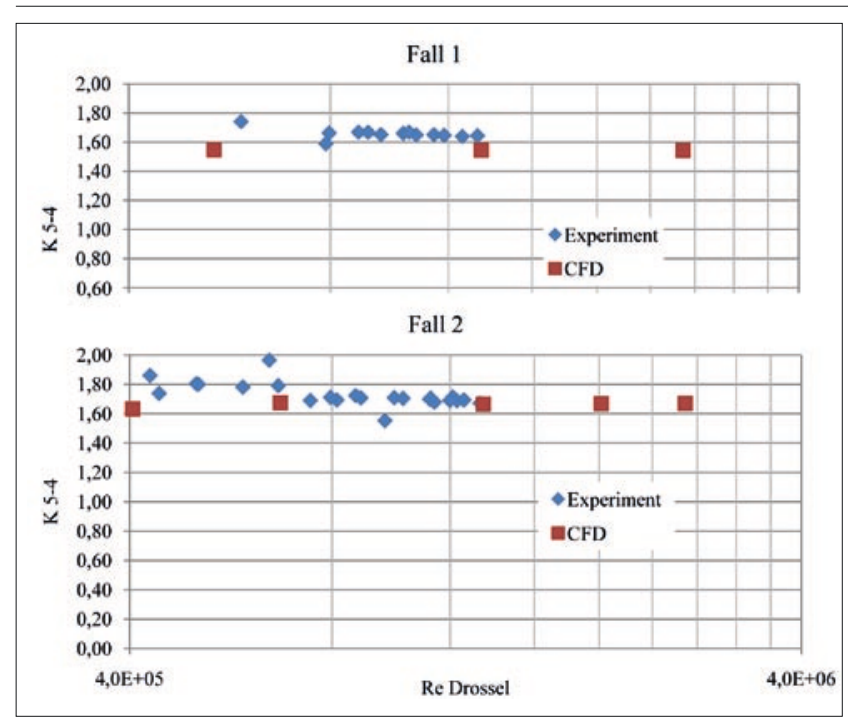

Abb. 8: Verlustbeiwerte K 5-4, Fall 1 und 2.

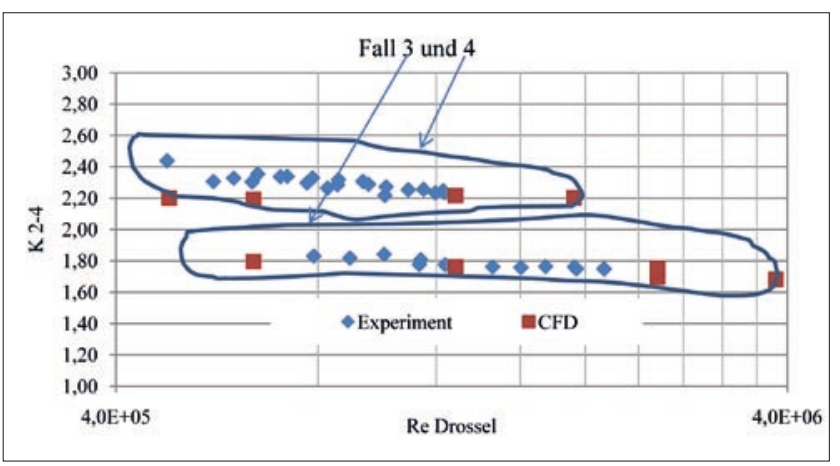

Abb. 9: Verlustbeiwerte K 2-4, Fall 3 und 4.

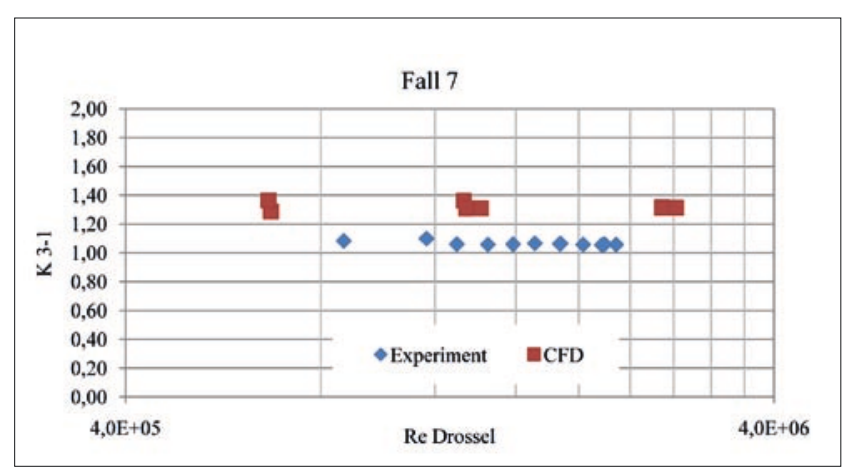

Abb. 10: Verlustbeiwerte K 3-1, Fall 7.

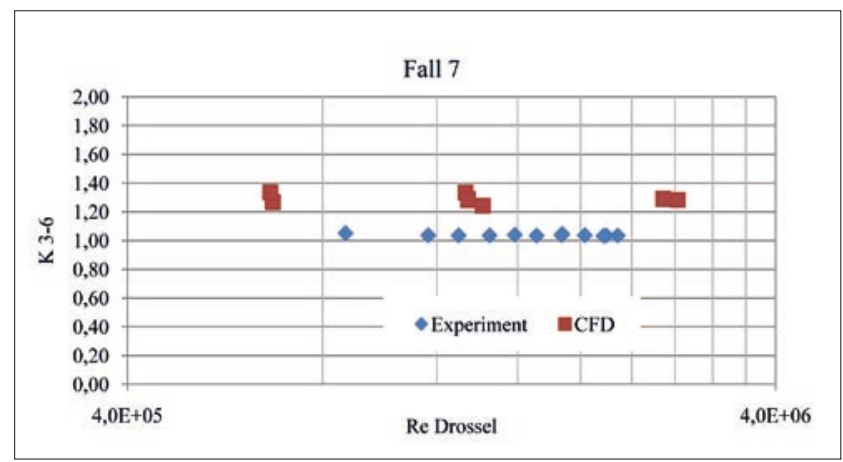

Abb. 11: Verlustbeiwerte K3-6, Fall 7.
Drücken addiert, um die totalen Drücke (= statischer Druck + dynamischer Druck) zu erhalten (Miller, 1978).

Je nach Lastfall betrugen die Durchflüsse zwischen $20 \mathrm{l} / \mathrm{s}$ und $90 \mathrm{l} / \mathrm{s}$ im Modellversuch. In allen Lastfällen war der Verlustbeiwert entweder - innerhalb der erzielbaren Messgenauigkeit - konstant, oder er näherte sich mit zunehmendem Durchfluss jeweils einem konstanten Wert an. Die Verlustbeiwerte wurden dann auf die Natur-Reynoldszahlen extrapoliert (vgl. z. B. Klasinc et al., 1992) und sind in Tabelle 2 zusammengefasst und werden später mit den Ergebnissen der CFD-Simulation verglichen.

\section{CFD-Simulation}

\subsection{Allgemeines}

Die CFD-Simulationen wurden mit Fluent 6.3.26 durchgeführt und die wandnahen Zonen in der Regel mit der "standard wall function" modelliert - die Größe der wandnahen Zellen lag im Be- reich von $2 \mathrm{~mm}$. Die Netz-Unabhängigkeit wurde anhand mehrerer Netze überprüft.

\subsection{Ergebnisse der CFD-Simulation}

Die CFD-Simulationen wurden für alle beschriebenen Lastfälle mit variierenden Durchflüssen durchgeführt. In den Fällen 1 bis 4 stimmten die Verlustbeiwerte mit den Experimenten sehr gut überein (siehe Abb. 8 und Abb. 9).

In den Fällen 5 bis 7 wichen die Ergebnisse der CFD-Simulation von den Experimenten ab: die Verluste waren in der Simulation um ca. $20 \%$ höher. In diesen Lastfällen trifft die aus der Drossel austretende Strömung direkt und in sehr hoher Geschwindigkeit auf die gegenüberliegende Wand des Krümmers - aufgrund der hohen Druck- bzw. Geschwindigkeitsgradienten erschien es sinnvoll, die Grenzschicht aufzulösen - was aber zu äußerst kleinen Zellen führt und aufgrund der hohen Rechenzeiten und des Speicherbedarfs kaum durchführbar ist. Ein paar Si- mulationen wurden mit aufwändigeren Turbulenzmodellen und mit sehr kleinen Zellen durchgeführt ( $\mathrm{y}^{+}$-Werte zwischen 2 und 10), jedoch konnte trotz des hohen Aufwandes der im Experiment beobachtete Verlustbeiwert nicht exakt nachgerechnet werden. Es scheint, dass die CFDSimulation hier an ihre Grenzen stößt.

Korrespondenz:

TU-Wien, Institut für Wasserbau und Ingenieurhydrologie Foschungsbereich Wasserbau

Karlsplatz 13/222

1040 Wien

E-Mail: boris.huber@kw.tuwien.ac.at

\section{LITERATUR}

Kobus H (1978) „Wasserbauliches Versuchswesen", Dt. Verband für Wasserwirtschaft, Mitteilungsheft 4

Miller DS (1978) “Internal Flow Systems”, BHRA Fluid Engineering, Vol.5

Klasinc R, Knoblauch H, Dum T (1992) „Power Losses in Distribution Pipes", 4th International Conference Hydrosoft 92, Valencia,

Fluent 6.3 manual 